\title{
The Application of Back-to-Back Accelerometers to Precision Vibration Measurements
}

\author{
B. F. Payne \\ National Bureau of Standards, Washington, DC 20234
}

Accepted: August 16, 1982

\begin{abstract}
Precision vibration measurements depend on accurate and repeatable calibration methods. Standardization of calibration test equipment and measurement techniques ensures more accurate and repeatable measurements. The use of the back-to-back accelerometer as a laboratory standard has become widespread. However, this use has been somewhat limited because of inadequate calibration methods. Recent developments in improved calibration methods have given the back-to-back accelerometer a greater potential as an accurate, repeatable, and stable vibration standard. As a vibration standard, the back-to-back accelerometer should prove to be a valuable asset for laboratories involved in vibration measurements and vibration transducer calibrations. By adapting existing techniques of laser interferometric calibration to the special geometry of the back-to-back accelerometer, improved accuracy (over existing methods) can be obtained over the range of $2-15,000 \mathrm{~Hz}$ and extension to $20,000 \mathrm{~Hz}$ is a good possibility. Recent work at NBS in this area is presented along with a description of a sample back-to-back transducer calibration.
\end{abstract}

Key words: accelerometers; calibration; exciters; shakers; standards; vibration.

\section{Introduction}

There is a widespread need for accurate vibration measurement systems in government and industrial laboratories. The back-to-back (BTB) accelerometer has certain advantages for use as a laboratory standard which will be discussed in this paper. It also has some unique problems with regard to its calibration. Some experimental data are presented for a sample BTB accelerometer utilizing two calibration techniques: an absolute calibration using an optical interferometer; and a comparison to another standard accelerometer.

\section{The Special Geometry of the BTB Accelerometer}

Figure 1 shows several typical BTB accelerometers photographed with their mounting bases pointing upward. The case of the accelerometer is designed so that another accelerometer can be mounted on top of

About the Author: B. F. Payne is a physicist with the NBS Center for Manufacturing Engineering. the BTB accelerometer. This permits the use of a small shaker (which does not require provision for mounting an accelerometer inside) to be used for
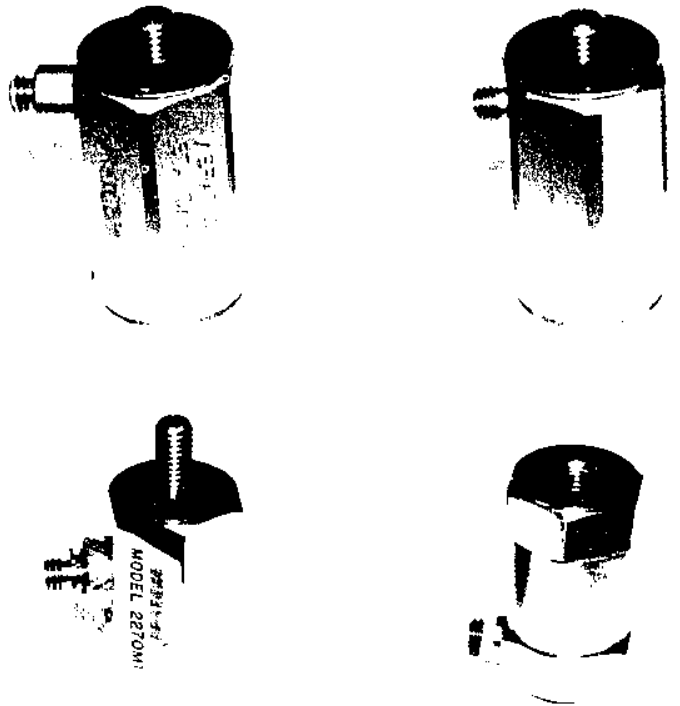

Figure 1-Typical back-to-back accelerometers. 
calibration, thus eliminating the need for a large shaker with removable fixtures for mounting a standard inside.

A BTB accelerometer mounted on a suitable shaker (fig. 2) can be used as a convenient system for comparison calibration of test accelerometers.

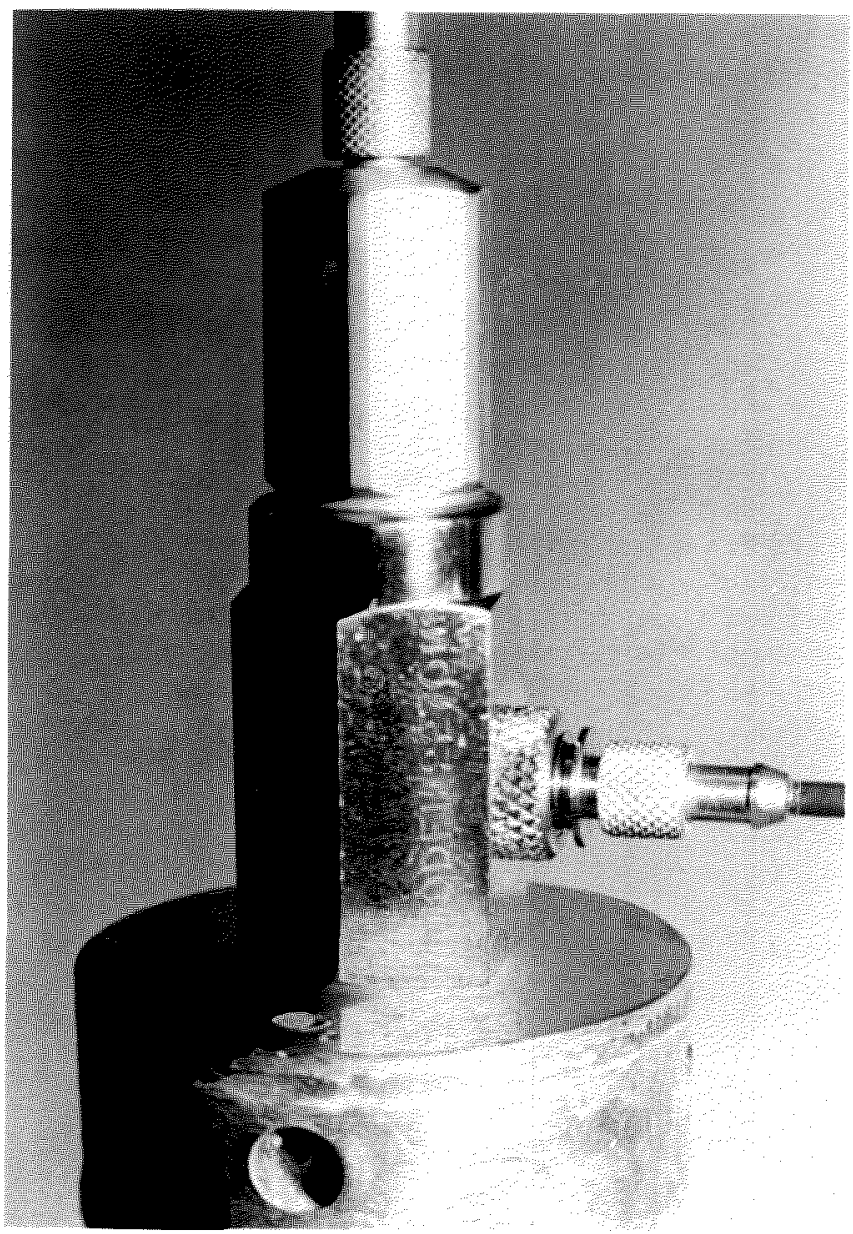

Figure 2-A back-to-back accelerometer with another accelerometer mounted on its top surface.

However, before the BTB accelerometer can be used to calibrate other accelerometers, the BTB accelerometer itself must first be accurately calibrated. One problem with this arrangement is that the BTB accelerometer is somewhat mass sensitive, especially above about $7 \mathrm{kHz}$. That is, the sensitivity of the BTB accelerometer is a function of the mass mounted on its top surface. Therefore, in order to obtain an accurate calibration, the BTB accelerometer must be calibrated with a mass on its top surface.

One approach is to calibrate the BTB accelerometer by using a calibrated single-ended accelerometer mounted on top of the BTB accelerometer (fig. 2). The top accelerometer can be calibrated by standard methods, with the BTB accelerometer calibrated by comparison to this accelerometer.

It is desirable, however, to use an absolute method for the calibration of the BTB accelerometer and thereby eliminate the two-step calibration procedure. The method developed at NBS uses a "dummy" mass fabricated of tungsten carbide, which has been lapped flat on both surfaces and has four small holes. The holes enable a laser light beam to pass through the mass and reflect from the BTB accelerometer's top surface, which has been polished to reflect light as a mirror. Utilizing this arrangement, an interferometric calibration is possible using the top surface of the BTB accelerometer as one of the interferometer mirrors $[1]^{1}$.

\subsection{Calibration of the BTB Accelerometer}

\section{Top Surface by Optical Interferometry}

A Michelson interferometer (fig. 3) is used to calibrate the BTB accelerometer [1]. Calibrations are obtained at each of four positions, corresponding to

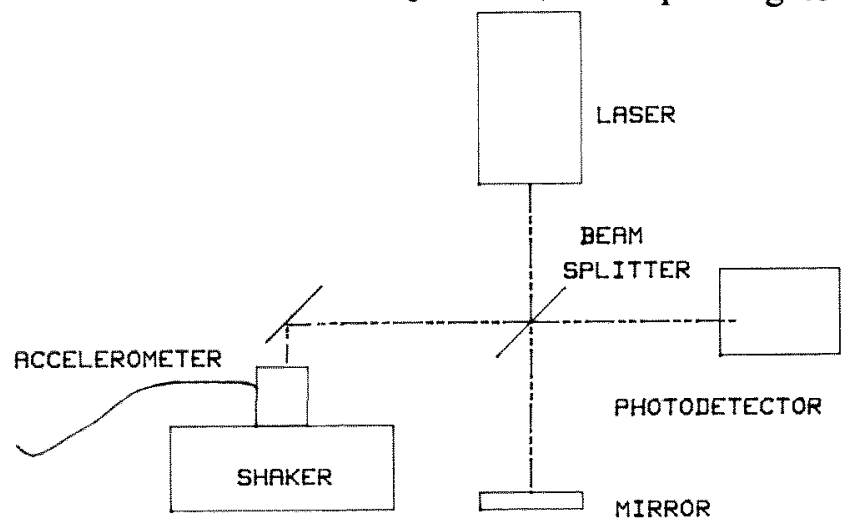

Figure 3-Michelson interferometer used for optical measurements.

the four holes in the dummy 19-gram mass shown in figure 4. Figure 5 shows the results of this calibration for the no-load and for the 19-gram average for the four positions for both the no-load and the 19-gramload conditions over the frequency range of $3-15 \mathrm{kHz}$. Figure 6 shows the average for the four positions for both the no-load and the 19-gram-load cases. The effect of the load is to decrease the sensitivity of the accelerometer. By averaging the sensitivities (corresponding to the four positions), a good representation of the actual sensitivity is obtained for the two cases. The data in figure 6 indicate that the effect of the load increases as the frequency increases. For frequencies below about $7 \mathrm{kHz}$ there is only a small mass loading effect whereas, for frequencies above about $7 \mathrm{kHz}$, the effect of the mass loading becomes more significant. Thus at $15 \mathrm{kHz}$, a

\footnotetext{
${ }^{1}$ Figures in brackets refer to the literature references at the end of this paper.
} 
calibration would be in error by about 2 percent if the effect of mass loading is not taken into account for a test accelerometer of about 19-gram mass.

\subsection{Calibration of the BTB Accelerometer by the Comparison Method}

The BTB accelerometer is calibrated by a comparison method in which a reference accelerometer (about 19 gram) is mounted on top of the BTB accelerometer (fig. 2). The reference accelerometer has been calibrated by comparison to NBS reference shakers (which have been reciprocity calibrated, 10-3500 $\mathrm{Hz}$ ) and by interferometric displacement measurement $(4000-10,000 \mathrm{~Hz})$ [2]. The comparison calibration is performed by energizing the shaker at a given test frequency, and measuring the voltage ratio of the output of the BTB accelerometer to the output of the reference accelerometer. This ratio is then multiplied by the sensitivity of the reference accelerometer, thereby yielding the sensitivity of the BTB accelerometer at this test frequency.

Similarly, calibrations are performed at the other test frequencies to obtain the complete calibration of the BTB accelerometer. The results of this comparison calibration of the BTB accelerometer are shown, together with the previously described absolute

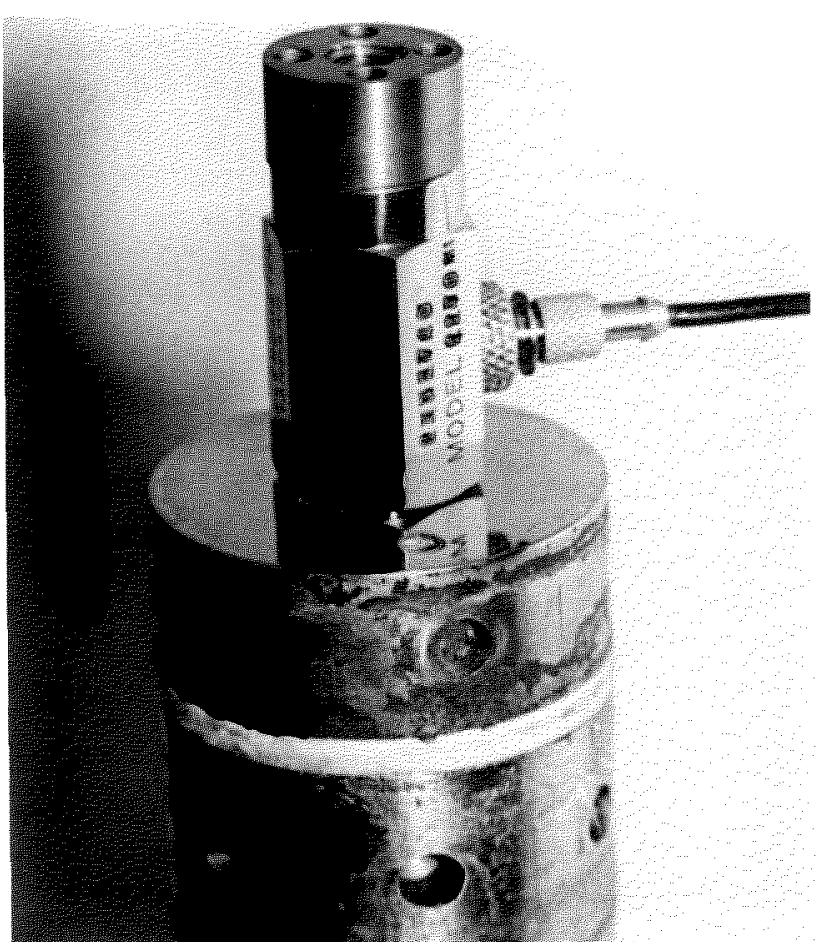

Figure 4-A back-to-back accelerometer with a dummy mass mounted for optical calibration.

calibration data, in figure 7. The data in figure 7 indicate agreement between the absolute interferometric method and the comparison method to
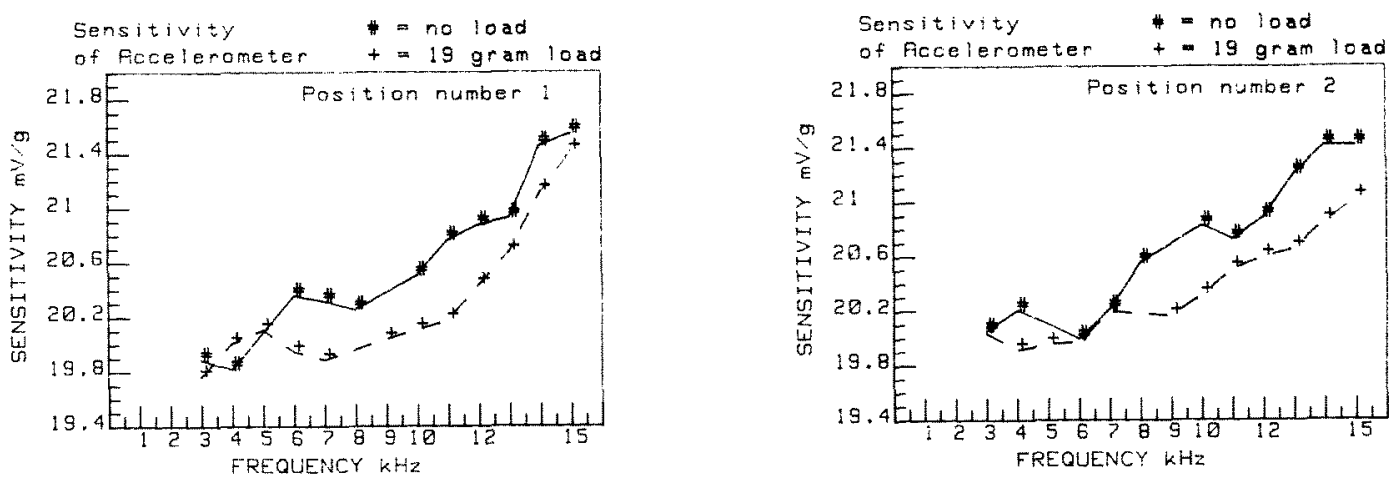

Figure 5-Optical calibration of a back-to-back accelerometer with no mass and 19-gram mass for four locations on the surface.
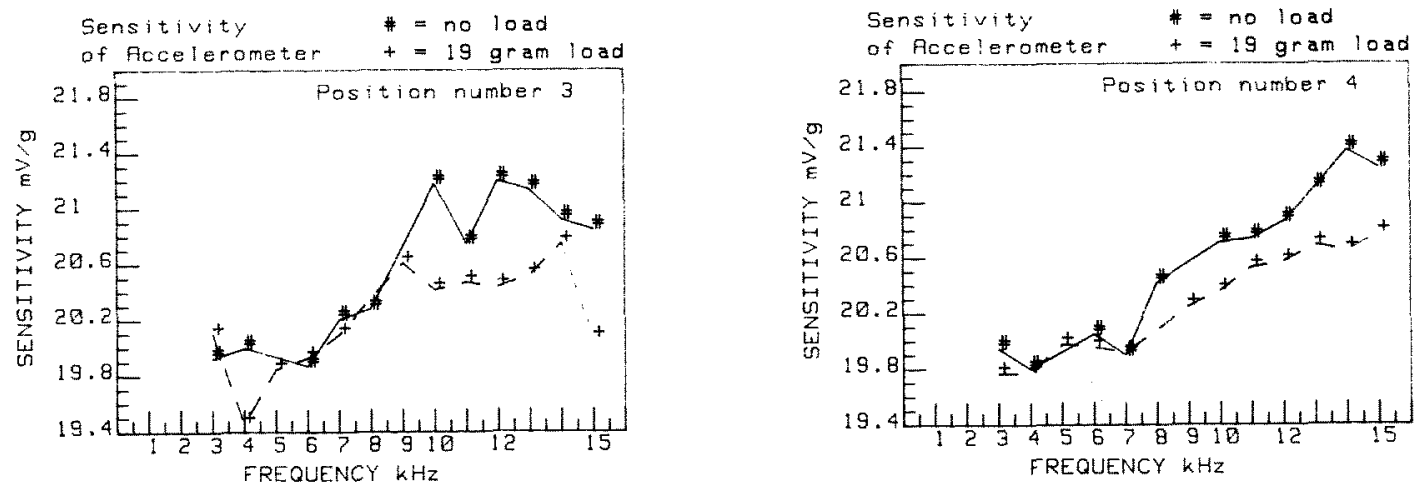


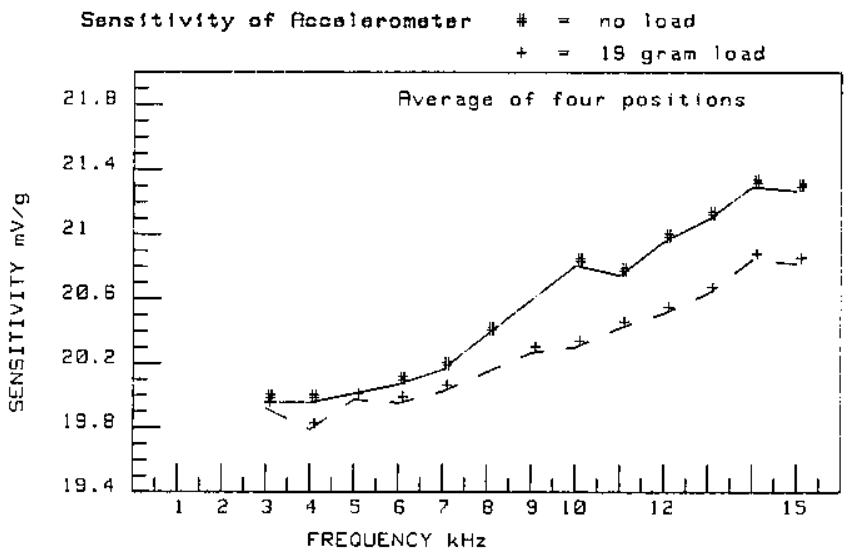

Figure 6-Optical calibration of a back-to-back accelerometer with no mass and 19-gram mass, average of four surface positions.

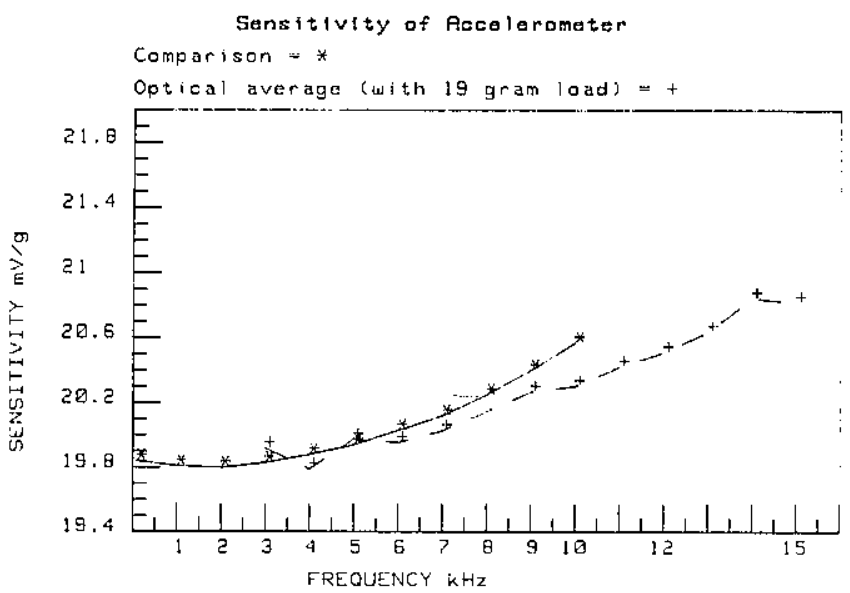

Figure 7-Optical and comparison calibration of a back-to-back accelerometer.

be within about 1 percent. Thus, the simple comparison method provides an accurate calibration of the BTB accelerometer, but does not yield the mass loading characteristics of the BTB accelerometer. One study [3] attempts to measure the mass loading by the comparison method by inserting masses between the BTB accelerometer and the reference accelerometer. This does give some qualitative results but does not take into account the relative motion between the accelerometers introduced by the inserted mass.

\section{The Use of the BTB Accelerometer as a Laboratory Standard}

Once calibrated, the BTB accelerometer, mounted on a suitable shaker, can be used as a reference standard, suitable for calibrating accelerometers up to a frequency of $15 \mathrm{kHz}$. The calibration is valid only for accelerometers of about 19-gram mass (since this is the calibration mass). However, this mass is typical of several commercial accelerometers in common use. Additional data need to be obtained to accommodate accelerometers of different masses. Work is being conducted at NBS to obtain such data by using a set of dummy masses to cover the range of interest and the results will be the subject of future publications.

\section{Conclusion}

The technique presented here eliminates one step in the calibration of a BTB accelerometer. Instead of using a two-step method of first calibrating a singleended accelerometer and then calibrating the BTB accelerometer by comparison to it, a one-step absolute method is used. It is desirable to have a standard calibrated by an absolute method in terms of fundamental units (e.g., the wavelength of light). Further data need to be collected on BTB accelerometers which are typically in use as laboratory standards. With these additional data, this technique will result in improved accuracy calibrations in the $3-$ to $15-\mathrm{kHz}$ frequency range. In addition to improved accuracy, the absolute method described above yields additional information about the accelerometer (mass loading characteristics) which the comparison method does not yield.

The BTB accelerometer, when properly calibrated under a loaded condition, can be an accurate and repeatable calibration standard. This standard, when mounted on a suitable shaker, will provide a convenient and accurate setup for performing comparison accelerometer calibrations.

\section{References}

[1] Payne, B. F. Absolute calibration of back-to-back accelerometers, Proceedings of the $27 \mathrm{th}$ international instrumentation symposium; 1981 May; Indianapolis, IN; 1981. 483-488.

[2] Payne, B. F; Koyanagi, R. S.; Federman, C.; Jones, E. Accelerometer calibration at the National Bureau of Standards, Proceedings of the 21st international instrumentation symposium ASD/TMD; 1975 May 19-21; Philadelphia, PA; 1975. 1-17.

[3] Koyanagi, R. S.; Pollard, J. D.; Ramboz, J. D. A systematic study of vibration transfer standards-mounting effects, Natl. Bur. Stand. (U.S.) NBSIR 73-291; 1973 September. 42p. 